\title{
Convergence and stability of the compensated split-step $\theta$-method for stochastic differential equations with jumps
}

\author{
Jianguo $\operatorname{Tan}^{1 *}$, Zhiming $\mathrm{Mu}^{2}$ and Yongfeng Guo
}

\footnotetext{
"Correspondence: tanjg@tju.edu.cn ${ }^{1}$ Department of Mathematics, Tianjin Polytechnic University, Tianjin, 300387, P.R. China Full list of author information is available at the end of the article
}

\begin{abstract}
In this paper, we develop a new compensated split-step $\theta(\operatorname{CSS} \theta)$ method for stochastic differential equations with jumps (SDEwJs). First, it is proved that the proposed method is convergent with strong order $1 / 2$ in the mean-square sense. Then the condition of the mean-square (MS) stability of the CSS $\theta$ method is obtained. Finally, some scalar test equations are simulated to verify the results obtained from theory, and a comparison between the compensated stochastic theta (CST) method by Wang and Gan (Appl. Numer. Math. 60:877-887, 2010) and CSS $\theta$ is analyzed. Meanwhile, the results show the higher efficiency of the $\operatorname{css} \theta$ method.
\end{abstract}

Keywords: stochastic differential equations; Poisson jumps; compensated split-step $\theta$-method; convergence; mean-square stability

\section{Introduction}

In this paper, we consider one-dimensional Itô stochastic differential equations (SDEs) with Poisson-driven jumps

$$
d X(t)=f\left(X\left(t^{-}\right)\right) d t+g\left(X\left(t^{-}\right)\right) d W(t)+h\left(X\left(t^{-}\right)\right) d N(t)
$$

for $t>0$, with $X\left(0^{-}\right)=X_{0}$, where $X\left(t^{-}\right)$denotes $\lim _{s \rightarrow t^{-}} X(s), f: \mathbb{R} \rightarrow \mathbb{R}, g: \mathbb{R} \rightarrow \mathbb{R}, h$ : $\mathbb{R} \rightarrow \mathbb{R}, W(t)$ is a scalar standard Wiener process, and $N(t)$ is a scalar Poisson process with intensity $\lambda$.

Recently, stochastic differential equations with jumps (SDEwJs) are becoming increasingly used to model real-world phenomena in different fields, such as economics, finance, biology, and physics. However, few analytical solutions have been proposed so far; thus, it is necessary to develop numerical methods for SDEwJs and study the properties of these methods. For example, Higham and Kloeden [1] studied the convergence and stability of the implicit method for jump-diffusion systems, and they further analyzed the strong convergence rates of the backward Euler method for a nonlinear jump-diffusion system [2]. Chalmers and Higham [3] studied the convergence and stability for the implicit simulations of SDEs with random jump magnitudes. Higham and Kloeden [4] constructed the split-step backward Euler (SSBE) method and the compensated split-step backward Euler (CSSBE) method for nonlinear SDEwJs. Bruti-Liberati and Platen $[5,6]$ developed strong and weak approximations of SDEwJs.

\section{Springer}

(2014 Tan et al.; licensee Springer. This is an Open Access article distributed under the terms of the Creative Commons Attribution License (http://creativecommons.org/licenses/by/2.0), which permits unrestricted use, distribution, and reproduction in any medium, provided the original work is properly cited. 
Lately, Wang and Gan [7] started to focus on the CST method for stochastic differential equations with jumps. Hu and Gan [8] studied the convergence and stability of the balanced methods for SDEwJs. The split-step $\theta$ (SS $\theta$ ) method was firstly developed by Ding et al. [9] to solve the stochastic differential equations. Thus, we will construct the compensated split-step $\theta$ method (CSS $\theta$ ) for SDEwJs.

In this paper, we investigate the convergence and mean-square stability of the CSS $\theta$ method for SDEwJs. The outline of the paper is as follows. In Section 2, we introduce some notations and hypotheses and give the $\operatorname{CSS} \theta$ method for SDEwJs. In Section 3, we prove that the numerical solutions produced by the $\operatorname{CSS} \theta$ method converge to the true solutions with strong order $1 / 2$. In Section 4, the mean-square stability of the $\operatorname{CSS} \theta$ method for linear test equation is studied. At last, some numerical experiments are used to verify the results obtained from the theory.

\section{The compensated split-step $\boldsymbol{\theta}$-method}

For the existence and uniqueness of the solution for (1.1), we usually assume that $f, g$, and $h$ satisfy the following assumptions:

(H1) (The uniform Lipschitz condition) There is a constant $K>0$, for all $x, y \in \mathbb{R}$, such that

$$
|f(x)-f(y)|^{2} \vee|g(x)-g(y)|^{2} \vee|h(x)-h(y)|^{2} \leq K|x-y|^{2} .
$$

(H2) (The linear growth condition) There is a constant $L>0$, for all $x \in \mathbb{R}$, such that

$$
|f(x)|^{2} \vee|g(x)|^{2} \vee|h(x)|^{2} \leq L\left(1+\left|x^{2}\right|\right) .
$$

We assume that the initial data $E|X(0)|^{2}$ is finite and $X(0)$ is independent of $W(t)$ and $N(t)$ for all $t \geq 0$. Under these conditions, we note that equation (1.1) has a unique solution on $[0,+\infty)$, see $[10,11]$.

For a constant step size $h=\Delta t>0$, we first define the split-step $\theta$ (SS $\theta)$ method for (1.1) by $Y_{0}=X\left(0^{-}\right)$and

$$
\begin{aligned}
& Y_{n}{ }^{*}=Y_{n}+\left[(1-\theta) f\left(Y_{n}\right)+\theta f\left(Y_{n}^{*}\right)\right] \Delta t, \\
& Y_{n+1}=Y_{n}{ }^{*}+g\left(Y_{n}{ }^{*}\right) \Delta W_{n}+h\left(Y_{n}^{*}\right) \Delta N_{n},
\end{aligned}
$$

where $\theta \in[0,1], Y_{n}$ is the numerical approximation of $X\left(t_{n}\right)$ with $t_{n}=n \cdot \Delta t$. Moreover, the increments $\Delta W_{n}:=W\left(t_{n+1}\right)-W\left(t_{n}\right)$ are independent Gaussian random variables with mean 0 and variance $\Delta t ; \Delta N_{n}:=N\left(t_{n+1}\right)-N\left(t_{n}\right)$ are independent Poisson distributed random variables with mean $\lambda \Delta t$ and variance $\lambda \Delta t$.

If we give $\theta=1$, the $\operatorname{SS} \theta$ method becomes the SSBE method in [4]. If $\theta=0$, the $\operatorname{SS} \theta$ method is an explicit method.

Note that the compensated Poisson process

$$
\tilde{N}(t):=N(t)-\lambda t
$$

which is a martingale. Defining

$$
f_{\lambda}:=f(x)+\lambda h(x)
$$


we can rewrite the jump-diffusion system (1.1) in the form

$$
\mathrm{d} X(t)=f_{\lambda}\left(X\left(t^{-}\right)\right) \mathrm{d} t+g\left(X\left(t^{-}\right)\right) \mathrm{d} W(t)+h\left(X\left(t^{-}\right)\right) \mathrm{d} \tilde{N}(t) .
$$

We note that $f_{\lambda}$ also satisfies the uniform Lipschitz condition and linear growth condition with larger constants

$$
K_{\lambda}=2(\lambda+1)^{2} K, \quad L_{\lambda}=2(\lambda+1)^{2} L
$$

Then we define the compensated split-step $\theta$ method $(\operatorname{CSS} \theta)$ for $(1.1)$ by $Y_{0}=X\left(0^{-}\right)$and

$$
\begin{aligned}
& Y_{n}{ }^{*}=Y_{n}+\left[(1-\theta) f_{\lambda}\left(Y_{n}\right)+\theta f_{\lambda}\left(Y_{n}^{*}\right)\right] \Delta t, \\
& Y_{n+1}=Y_{n}{ }^{*}+g\left(Y_{n}^{*}\right) \Delta W_{n}+h\left(Y_{n}^{*}\right) \Delta \tilde{N}_{n},
\end{aligned}
$$

where $\Delta \tilde{N}_{n}:=\tilde{N}\left(t_{n+1}\right)-\tilde{N}\left(t_{n}\right)$.

If we give $\theta=1$, the $\operatorname{CSS} \theta$ method becomes the CSSBE method in [4].

To answer the question of the existence of numerical solution, we will give the following lemma.

Lemma 2.1 Assume that $f: \mathbb{R} \rightarrow \mathbb{R}$ satisfies (2.1), and let $0<\theta<1,0<\Delta t<1 /\left(\sqrt{K_{\lambda}} \theta\right)$, then equation (2.7) can be solved uniquely for $Y_{n}{ }^{*}$, with probability 1 .

Proof Writing (2.7) as $Y_{n}{ }^{*}=F\left(Y_{n}{ }^{*}\right)=a+\theta \Delta t f_{\lambda}\left(Y_{n}{ }^{*}\right), a \in \mathbb{R}$, and using condition (2.6), we have

$$
\begin{aligned}
|F(u)-F(v)| & =\left|\theta \Delta t f_{\lambda}(u)-\theta \Delta t f_{\lambda}(v)\right| \\
& \leq \sqrt{K_{\lambda}} \theta \Delta t|u-v| .
\end{aligned}
$$

Then the result follows from the classical Banach contraction mapping theorem [12].

\section{Strong convergence on a finite time interval $[0, T]$}

In this section, we prove the strong convergence of the $\operatorname{CSS} \theta$ method for problem (1.1) on a finite time interval $[0, T]$, where $T$ is a constant.

When Lemma 2.1 is followed, we find it is convenient to use continuous-time approximation solution in our strong convergence analysis. Hence, for $t \in\left[t_{n}, t_{n+1}\right)$, we can define the two step-functions:

$$
\begin{aligned}
& Z_{1}(t)=\sum_{n=0}^{N-1} Y_{n} I_{[n \Delta t,(n+1) \Delta t)}(t), \\
& Z_{2}(t)=\sum_{n=0}^{N-1} Y_{n}^{*} I_{[n \Delta t,(n+1) \Delta t)}(t),
\end{aligned}
$$

where $N$ is the largest number such that $N \Delta t \leq T$, and $I_{A}$ is the indicator function for the set $A$, i.e., $I_{A}(x)= \begin{cases}1, & x \in A, \\ 0, & x \notin A .\end{cases}$ 
When $t \in\left[t_{n}, t_{n+1}\right)$, Lemma 2.1 ensures the existence of $Y_{n}^{*}$ by (2.7), then we define

$$
\begin{aligned}
Y(t)= & Y_{n}+\left[(1-\theta) f_{\lambda}\left(Y_{n}\right)+\theta f_{\lambda}\left(Y_{n}^{*}\right)\right]\left(t-t_{n}\right)+g\left(Y_{n}^{*}\right)\left(W(t)-W\left(t_{n}\right)\right) \\
& +h\left(Y_{n}^{*}\right)\left(\tilde{N}(t)-\tilde{N}\left(t_{n}\right)\right) .
\end{aligned}
$$

Thus we can rewrite (3.3) in the integral form as follows:

$$
\begin{aligned}
Y(t)= & Y_{0}+\int_{0}^{t}(1-\theta) f_{\lambda}\left(Z_{1}(s)\right)+\theta f_{\lambda}\left(Z_{2}(s)\right) \mathrm{d} s+\int_{0}^{t} g\left(Z_{2}(s)\right) \mathrm{d} W(s) \\
& +\int_{0}^{t} h\left(Z_{2}(s)\right) \mathrm{d} \tilde{N}(s) .
\end{aligned}
$$

It is easy to verify that $Z_{1}\left(t_{n}\right)=Y_{n}=Y\left(t_{n}\right)$, that is, $Z_{1}(t)$ and $Y(t)$ coincide with the discrete solutions at the gridpoints. Hence we refer to $Y(t)$ as a continuous-time extension of the discrete approximation $\left\{Y_{n}\right\}$. So our plan is to prove a strong convergence result for $Y(t)$.

Now we begin the proof of the strong convergence of the $\operatorname{CSS} \theta$ method, our first lemma shows the relationship between $E\left|Y_{n}{ }^{*}\right|^{2}$ and $E\left|Y_{n}\right|^{2}$.

Lemma 3.1 Suppose $f: \mathbb{R} \rightarrow \mathbb{R}$ satisfies (2.2), and let $0<\theta<1,0<\Delta t<\min \left\{1, \frac{1}{4 \theta L_{\lambda}}\right\}$, then there exist two positive constants $A=4\left(1+L_{\lambda}\right)$ and $B=8 L_{\lambda}$ such that

$$
E\left|Y_{n}^{*}\right|^{2} \leq A E\left|Y_{n}\right|^{2}+B
$$

where $Y_{n}^{*}$ and $Y_{n}$ are produced by (2.7) and (2.8).

Proof Squaring both sides of (2.7), we find

$$
\begin{aligned}
\left|Y_{n}{ }^{*}\right|^{2}= & \left|Y_{n}+(1-\theta) \Delta t f_{\lambda}\left(Y_{n}\right)+\theta \Delta t f_{\lambda}\left(Y_{n}{ }^{*}\right)\right|^{2} \\
= & \left|Y_{n}\right|^{2}+\left|(1-\theta) \Delta t f_{\lambda}\left(Y_{n}\right)\right|^{2}+\left|\theta \Delta t f_{\lambda}\left(Y_{n}^{*}\right)\right|^{2}+2 \theta \Delta t f_{\lambda}\left(Y_{n}{ }^{*}\right) Y_{n} \\
& +2(1-\theta) \Delta t f_{\lambda}\left(Y_{n}\right) Y_{n}+2 \theta(1-\theta) \Delta t^{2} f_{\lambda}\left(Y_{n}\right) f_{\lambda}\left(Y_{n}{ }^{*}\right) .
\end{aligned}
$$

Using the elementary inequality $2 a b \leq a^{2}+b^{2}$, we obtain

$$
\begin{aligned}
\left|Y_{n}{ }^{*}\right|^{2} \leq & \left|Y_{n}\right|^{2}+(1-\theta)^{2} \Delta t^{2}\left|f_{\lambda}\left(Y_{n}\right)\right|^{2}+\theta^{2} \Delta t^{2}\left|f_{\lambda}\left(Y_{n}{ }^{*}\right)\right|^{2} \\
& +\theta \Delta t\left[\left|Y_{n}\right|^{2}+\left|f_{\lambda}\left(Y_{n}{ }^{*}\right)\right|^{2}\right]+(1-\theta) \Delta t\left[\left|Y_{n}\right|^{2}+\left|f_{\lambda}\left(Y_{n}\right)\right|^{2}\right] \\
& +\theta(1-\theta) \Delta t^{2}\left[\left|f_{\lambda}\left(Y_{n}\right)\right|^{2}+\left|f_{\lambda}\left(Y_{n}{ }^{*}\right)\right|^{2}\right] \\
= & \left|Y_{n}\right|^{2}+\left[(1-\theta)^{2} \Delta t^{2}+(1-\theta) \Delta t+\theta(1-\theta) \Delta t^{2}\right]\left|f_{\lambda}\left(Y_{n}\right)\right|^{2} \\
& +\Delta t\left|Y_{n}\right|^{2}+\left[\theta^{2} \Delta t^{2}+\theta \Delta t+\theta(1-\theta) \Delta t^{2}\right]\left|f_{\lambda}\left(Y_{n}{ }^{*}\right)\right|^{2} \\
= & \left|Y_{n}\right|^{2}+\left[(1-\theta) \Delta t^{2}+(1-\theta) \Delta t\right]\left|f_{\lambda}\left(Y_{n}\right)\right|^{2} \\
& +\Delta t\left|Y_{n}\right|^{2}+\left[\theta \Delta t^{2}+\theta \Delta t\right]\left|f_{\lambda}\left(Y_{n}{ }^{*}\right)\right|^{2} .
\end{aligned}
$$


Due to $\Delta t<1$, linear growth condition (2.6), and $0<\theta<1$, we can get

$$
\begin{aligned}
\left|Y_{n}{ }^{*}\right|^{2} \leq & \left|Y_{n}\right|^{2}+2(1-\theta) \Delta t L_{\lambda}\left(1+\left|Y_{n}\right|^{2}\right)+\Delta t\left|Y_{n}\right|^{2} \\
& +2 \theta \Delta t L_{\lambda}\left(1+\left|Y_{n}{ }^{*}\right|^{2}\right) \\
\leq & \left|Y_{n}\right|^{2}+2(1-\theta) \Delta t L_{\lambda}\left|Y_{n}\right|^{2}+\Delta t\left|Y_{n}\right|^{2} \\
& +2 \theta \Delta t L_{\lambda}\left|Y_{n}{ }^{*}\right|^{2}+2\left(L_{\lambda}+L_{\lambda}\right) \Delta t .
\end{aligned}
$$

Taking mathematical expectation for both sides, we can obtain

$$
\begin{aligned}
E\left|Y_{n}{ }^{*}\right|^{2} \leq & \left(1+2(1-\theta) \Delta t L_{\lambda}+\Delta t\right) E\left|Y_{n}\right|^{2} \\
& +2 \theta \Delta t L_{\lambda} E\left|Y_{n}{ }^{*}\right|^{2}+4 L_{\lambda} \Delta t .
\end{aligned}
$$

Since $2 \theta L_{\lambda} \Delta t<1 / 2$, thus $1-2 \theta L_{\lambda} \Delta t \geq 1 / 2$, then by $\Delta t<1$ and $0<\theta<1$, we have

$$
\begin{aligned}
E\left|Y_{n}{ }^{*}\right|^{2} & \leq \frac{\left(1+2(1-\theta) \Delta t L_{\lambda}+\Delta t\right)}{1-2 \theta L_{\lambda} \Delta t} E\left|Y_{n}\right|^{2}+\frac{4 L_{\lambda} \Delta t}{1-2 \theta \Delta t L_{\lambda}} \\
& \leq 2\left(1+2 L_{\lambda}+1\right) E\left|Y_{n}\right|^{2}+8 L_{\lambda} \\
& =A E\left|Y_{n}\right|^{2}+B
\end{aligned}
$$

where $A=4\left(1+L_{\lambda}\right)$ and $B=8 L_{\lambda}$. The proof is completed.

The next lemma shows that the discrete numerical solutions $Y_{n}$ and $Y_{n}{ }^{*}(n=0,1, \ldots, N)$, produced by the $\operatorname{CSS} \theta$ method, have bounded second moments.

Lemma 3.2 Under conditions (2.1)-(2.2), let $Y_{n}$ and $Y_{n}{ }^{*}(n=0,1, \ldots, N)$ be produced by (2.7) and (2.8), and let $0<\theta<1,0<\Delta t<\min \left\{1, \frac{1}{4 \theta L_{\lambda}}, \frac{1}{\sqrt{K_{\lambda}} \theta}\right\}$, then

$$
E\left|Y_{n}\right|^{2} \leq C_{1}
$$

and

$$
E\left|Y_{n}{ }^{*}\right|^{2} \leq C_{2}
$$

where $C_{1}$ and $C_{2}$ are two positive constants independent of $\Delta t$.

Proof By Lemma 2.1, we can express the CSS $\theta$ method (2.7) and (2.8) in the following form:

$$
\begin{aligned}
Y_{n+1}= & Y_{0}+\int_{0}^{(n+1) \Delta t}\left[(1-\theta) f_{\lambda}\left(Z_{1}(s)\right)+\theta f_{\lambda}\left(Z_{2}(s)\right)\right] \mathrm{d} s \\
& +\int_{0}^{(n+1) \Delta t} g\left(Z_{2}(s)\right) \mathrm{d} W(s)+\int_{0}^{(n+1) \Delta t} h\left(Z_{2}(s)\right) \mathrm{d} \tilde{N}(s),
\end{aligned}
$$

where $n=0,1, \ldots, N-1$. 
Squaring both sides, taking the mathematical expectation and using the element inequality $(a+b+c+d)^{2} \leq 4|a|^{2}+4|b|^{2}+4|c|^{2}+4|d|^{2}$, we have

$$
\begin{aligned}
E\left|Y_{n+1}\right|^{2} \leq & 4 E\left|Y_{0}\right|^{2}+4 E\left|\int_{0}^{(n+1) \Delta t}\left[(1-\theta) f_{\lambda}\left(Z_{1}(s)\right)+\theta f_{\lambda}\left(Z_{2}(s)\right)\right] \mathrm{d} s\right|^{2} \\
& +4 E\left|\int_{0}^{(n+1) \Delta t} g\left(Z_{2}(s)\right) \mathrm{d} W(s)\right|^{2} \\
& +4 E\left|\int_{0}^{(n+1) \Delta t} h\left(Z_{2}(s)\right) \mathrm{d} \tilde{N}(s)\right|^{2}
\end{aligned}
$$

Now, using the Cauchy-Schwarz inequality and the inequality $|\theta x+(1-\theta) y|^{2} \leq \theta|x|^{2}+$ $(1-\theta)|y|^{2}$, the linear growth condition (2.6) and Fubini's theorem, we can get

$$
\begin{aligned}
& E\left|\int_{0}^{(n+1) \Delta t}\left[(1-\theta) f\left(Z_{1}(s)\right)+\theta f\left(Z_{2}(s)\right)\right] \mathrm{d} s\right|^{2} \\
& \quad \leq T E \int_{0}^{(n+1) \Delta t}\left|(1-\theta) f_{\lambda}\left(Z_{1}(s)\right)+\theta f_{\lambda}\left(Z_{2}(s)\right)\right|^{2} \mathrm{~d} s \\
& \quad \leq 2 T E \int_{0}^{(n+1) \Delta t}\left|f_{\lambda}\left(Z_{1}(s)\right)\right|^{2}+\left|f_{\lambda}\left(Z_{2}(s)\right)\right|^{2} \mathrm{~d} s \\
& \quad \leq 2 T L_{\lambda} E \int_{0}^{(n+1) \Delta t} 2+\left|Z_{1}(s)\right|^{2}+\left|Z_{2}(s)\right|^{2} \mathrm{~d} s \\
& \quad \leq 4 T^{2} L_{\lambda}+2 T L_{\lambda} \int_{0}^{(n+1) \Delta t} E\left|Z_{1}(s)\right|^{2}+E\left|Z_{2}(s)\right|^{2} \mathrm{~d} s \\
& \quad \leq 4 T^{2} L_{\lambda}+2 T L_{\lambda} \Delta t\left(\sum_{i=0}^{n} E\left|Y_{i}\right|^{2}+\sum_{i=0}^{n} E\left|Y_{i}^{*}\right|^{2}\right) .
\end{aligned}
$$

Using the martingale isometry and linear growth condition (2.2), we have

$$
\begin{aligned}
E\left|\int_{0}^{(n+1) \Delta t} g\left(Z_{2}(s)\right) \mathrm{d} W(s)\right|^{2} & =\int_{0}^{(n+1) \Delta t} E\left|g\left(Z_{2}(s)\right)\right|^{2} \mathrm{~d} s \\
& =\Delta t \sum_{i=0}^{n} E\left|g\left(Y_{i}^{*}\right)\right|^{2} \\
& \leq \Delta t L \sum_{i=0}^{n}\left(1+E\left|Y_{i}^{*}\right|^{2}\right) \\
& \leq L T+\Delta t L \sum_{i=0}^{n} E\left|Y_{i}^{*}\right|^{2}
\end{aligned}
$$

For the jump integral, as the compensated Poisson process $\tilde{N}(t)=N(t)-\lambda t$ is a martingale, so we use the isometry

$$
E\left|\int_{a}^{b} h\left(Z_{2}(s)\right) \mathrm{d} \tilde{N}(s)\right|^{2}=\lambda \int_{a}^{b} E\left|h\left(Z_{2}(s)\right)\right|^{2} \mathrm{~d} s
$$


(see, for example, [13]), then we have

$$
\begin{aligned}
E\left|\int_{0}^{(n+1) \Delta t} h\left(Z_{2}(s)\right) \mathrm{d} \tilde{N}(s)\right|^{2} & =\lambda \int_{0}^{(n+1) \Delta t} E\left|h\left(Z_{2}(s)\right)\right|^{2} \mathrm{~d} s \\
& =\lambda \Delta t \sum_{i=0}^{n} E\left|h\left(Y_{i}^{*}\right)\right|^{2} \\
& \leq \lambda \Delta t L \sum_{i=0}^{n}\left(1+E\left|Y_{i}^{*}\right|^{2}\right) \\
& \leq \lambda T L+\lambda \Delta t L \sum_{i=0}^{n} E\left|Y_{i}^{*}\right|^{2} .
\end{aligned}
$$

Inserting (3.13)-(3.15) in (3.12) gives

$$
\begin{aligned}
E\left|Y_{n+1}\right|^{2} \leq & 4\left(E\left|Y_{0}\right|^{2}+4 T^{2} L_{\lambda}+L T+\lambda T L\right) \\
& +4 \Delta t\left(2 T L_{\lambda}+L+\lambda L\right) \sum_{i=0}^{n} E\left|Y_{i}^{*}\right|^{2} \\
& +8 T L_{\lambda} \Delta t \sum_{i=0}^{n} E\left|Y_{i}\right|^{2} .
\end{aligned}
$$

By Lemma 3.1, we can derive that

$$
\begin{aligned}
E\left|Y_{n+1}\right|^{2} \leq & 4\left(E\left|Y_{0}\right|^{2}+4 T^{2} L_{\lambda}+L T+\lambda T L\right) \\
& +4 \Delta t\left(2 T L_{\lambda}+L+\lambda L\right)\left(A \sum_{i=0}^{n} E\left|Y_{i}\right|^{2}+(n+1) B\right) \\
& +8 T L_{\lambda} \Delta t \sum_{i=0}^{n} E\left|Y_{i}\right|^{2} \\
\leq & 4\left(E\left|Y_{0}\right|^{2}+4 T^{2} L_{\lambda}+L T+\lambda T L\right) \\
& +4(n+1) B\left(2 T L_{\lambda}+L+\lambda L\right) \Delta t \\
& +\left[4 A\left(2 T L_{\lambda}+L+\lambda L\right)+8 T L_{\lambda}\right] \Delta t \sum_{i=0}^{n} E\left|Y_{i}\right|^{2} \\
\leq & c_{1}+c_{2} \Delta t \sum_{i=0}^{n} E\left|Y_{i}\right|^{2},
\end{aligned}
$$

where

$$
c_{1}=4\left(E\left|Y_{0}\right|^{2}+4 T^{2} L_{\lambda}+L T+\lambda T L\right)+4(n+1) B\left(2 T L_{\lambda}+L+\lambda L\right)
$$

and

$$
c_{2}=4 A\left(2 T L_{\lambda}+L+\lambda L\right)+8 T L_{\lambda}
$$

are both independent of $\Delta t$. 
Then, using the discrete Gronwall inequality, we can get

$$
E\left|Y_{n}\right|^{2} \leq c_{1} e^{c_{2}} \equiv C_{1}
$$

Then, by Lemma 3.1, we can obtain that

$$
E\left|Y_{n}{ }^{*}\right|^{2} \leq A E\left|Y_{n}\right|^{2}+B \leq A C_{1}+B \equiv C_{2} .
$$

The next lemma shows that the continuous-time approximation $Y(t)$ in (3.4) remains close to the step functions $Z_{1}(t)$ and $Z_{2}(t)$ in the mean square sense.

Lemma 3.3 Under conditions (2.1)-(2.2), let $Y_{n}{ }^{*}$ and $Y_{n}$ be produced by (2.7) and (2.8), and let $0<\theta<1,0<\Delta t<\min \left\{1, \frac{1}{4 \theta L_{\lambda}}, \frac{1}{\sqrt{K_{\lambda} \theta}}\right\}$, then there exist two positive constants $C_{3}$ and $C_{4}$ that are independent of $\Delta t$, such that

$$
E\left|Y(t)-Z_{1}(t)\right|^{2} \leq C_{3} \Delta t
$$

and

$$
E\left|Y(t)-Z_{2}(t)\right|^{2} \leq C_{4} \Delta t
$$

where $t \in[0, T], Z_{1}(t), Z_{2}(t)$, and $Y(t)$ are defined by (3.1), (3.2), (3.4), respectively.

Proof For any $t \in[0, T]$, there exists a nonnegative integer $n$ such that

$$
t \in[n \Delta t,(n+1) \Delta t] \subseteq[0, T]
$$

we have

$$
\begin{aligned}
Y(t)-Z_{1}(t)= & Y(t)-Y_{n} \\
= & \int_{n \Delta t}^{t}(1-\theta) f_{\lambda}\left(Z_{1}(s)\right)+\theta f_{\lambda}\left(Z_{2}(s)\right) \mathrm{d} s \\
& +\int_{n \Delta t}^{t} g\left(Z_{2}(s)\right) \mathrm{d} W(s) \\
& +\int_{n \Delta t}^{t} h\left(Z_{2}(s)\right) \mathrm{d} \tilde{N}(s) .
\end{aligned}
$$

Squaring both sides and using the element inequality $(a+b+c)^{2} \leq 3|a|^{2}+3|b|^{2}+3|c|^{2}$, we have

$$
\begin{aligned}
\left|Y(t)-Z_{1}(t)\right|^{2} \leq & 3\left|\int_{n \Delta t}^{t}\left[(1-\theta) f_{\lambda}\left(Z_{1}(s)\right)+\theta f_{\lambda}\left(Z_{2}(s)\right)\right] \mathrm{d} s\right|^{2} \\
& +3\left|\int_{n \Delta t}^{t} g\left(Z_{2}(s)\right) \mathrm{d} W(s)\right|^{2} \\
& +3\left|\int_{n \Delta t}^{t} h\left(Z_{2}(s)\right) \mathrm{d} \tilde{N}(s)\right|^{2}
\end{aligned}
$$


Taking mathematical expectation, by the element inequality $(a+b)^{2} \leq 2|a|^{2}+2|b|^{2}$, and using the martingale isometry, we have

$$
\begin{aligned}
E\left|Y(t)-Z_{1}(t)\right|^{2} \leq & 6 \Delta t \int_{n \Delta t}^{t}\left[E\left|f_{\lambda}\left(Z_{1}(s)\right)\right|^{2}+E\left|f_{\lambda}\left(Z_{2}(s)\right)\right|^{2}\right] \mathrm{d} s \\
& +3 \int_{n \Delta t}^{t} E\left|g\left(Z_{2}(s)\right)\right|^{2} \mathrm{~d} s \\
& +3 \lambda \int_{n \Delta t}^{t} E\left|h\left(Z_{2}(s)\right)\right|^{2} \mathrm{~d} s .
\end{aligned}
$$

By the linear growth conditions (2.2) and (2.6), we get

$$
\begin{aligned}
E\left|Y(t)-Z_{1}(t)\right|^{2} \leq & 6 \Delta t L_{\lambda} \int_{n \Delta t}^{t} 2+E\left|Z_{1}(s)\right|^{2}+E\left|Z_{2}(s)\right|^{2} \mathrm{~d} s \\
& +3 L(1+\lambda) \int_{n \Delta t}^{t} 1+E\left|Z_{2}(s)\right|^{2} \mathrm{~d} s .
\end{aligned}
$$

Since $Z_{1}(t) \equiv Y_{n}$ and $Z_{2}(t) \equiv Y_{n}^{*}$ on $[n \Delta t,(n+1) \Delta t)$, we have

$$
\begin{aligned}
E\left|Y(t)-Z_{1}(t)\right|^{2} \leq & 6 \Delta t^{2} L_{\lambda}\left(2+E\left|Y_{n}\right|^{2}+E\left|Y_{n}^{*}\right|^{2}\right) \\
& +3 L \Delta t(1+\lambda)\left(1+E\left|Y_{n}^{*}\right|^{2}\right) .
\end{aligned}
$$

Then, for each $t \in[0, T]$, and by Lemma 3.2, we can derive

$$
\begin{aligned}
E\left|Y(t)-Z_{1}(t)\right|^{2} \leq & 6 \Delta t^{2} L_{\lambda}\left(2+C_{1}+C_{2}\right) \\
& +3 L \Delta t(1+\lambda)\left(1+C_{2}\right) \\
\leq & C_{3} \Delta t
\end{aligned}
$$

where $C_{3}=6 L_{\lambda}\left(2+C_{1}+C_{2}\right)+3 L(1+\lambda)\left(1+C_{2}\right)$. Thus we can prove (3.18).

Now we give the proof of (3.19).

By (2.7) and for each $t \in[n \Delta t,(n+1) \Delta t] \subseteq[0, T]$, we get

$$
Z_{1}(t)-Z_{2}(t)=Y_{n}-Y_{n}{ }^{*}=-\left[(1-\theta) f_{\lambda}\left(Y_{n}\right)+\theta f_{\lambda}\left(Y_{n}{ }^{*}\right)\right] \Delta t .
$$

Using the inequality $|\theta x+(1-\theta) y|^{2} \leq \theta|x|^{2}+(1-\theta)|y|^{2}$, and $0<\theta<1$, we can get

$$
\begin{aligned}
\left|Z_{1}(t)-Z_{2}(t)\right|^{2} & =\left|(1-\theta) f_{\lambda}\left(Y_{n}\right)+\theta f_{\lambda}\left(Y_{n}{ }^{*}\right)\right|^{2} \Delta t^{2} \\
& \leq\left[(1-\theta)\left|f_{\lambda}\left(Y_{n}\right)\right|^{2}+\theta\left|f_{\lambda}\left(Y_{n}{ }^{*}\right)\right|^{2}\right] \Delta t^{2} \\
& \leq\left[\left|f_{\lambda}\left(Y_{n}\right)\right|^{2}+\left|f_{\lambda}\left(Y_{n}{ }^{*}\right)\right|^{2}\right] \Delta t^{2} .
\end{aligned}
$$

Taking mathematical expectation, and by the linear growth condition (2.6),

$$
\begin{aligned}
E\left|Z_{1}(t)-Z_{2}(t)\right|^{2} & \leq\left[E\left|f_{\lambda}\left(Y_{n}\right)\right|^{2}+E\left|f_{\lambda}\left(Y_{n}^{*}\right)\right|^{2}\right] \Delta t^{2} \\
& \leq L_{\lambda}\left(2+E\left|Y_{n}\right|^{2}+E\left|Y_{n}^{*}\right|^{2}\right) \Delta t^{2}
\end{aligned}
$$


Then by Lemma 3.2 we can derive

$$
E\left|Z_{1}(t)-Z_{2}(t)\right|^{2} \leq L_{\lambda}\left(2+C_{1}+C_{2}\right) \Delta t
$$

Then, by the element inequality $(a+b)^{2} \leq 2|a|^{2}+2|b|^{2}$ and using (3.20) and (3.21), we have

$$
\begin{aligned}
E\left|Y(t)-Z_{2}(t)\right|^{2} & \leq 2 E\left|Y(t)-Z_{1}(t)\right|^{2}+2 E\left|Z_{1}(t)-Z_{2}(t)\right|^{2} \\
& \leq 2 C_{3} \Delta t+2 L_{\lambda}\left(2+C_{1}+C_{2}\right) \Delta t \\
& \leq C_{4} \Delta t,
\end{aligned}
$$

where $C_{4}=2 C_{3}+2 L_{\lambda}\left(2+C_{1}+C_{2}\right)$. Then we have proved (3.19).

Now we use the above lemmas to prove a strong convergence result.

Definition 3.1 A numerical method is said to have strong order of convergence equal to $\gamma$ if there exists a constant $C$ such that the numerical solution sequence $Y_{n}$ produced by this numerical scheme satisfies

$$
E\left|Y_{n}-X(\tau)\right| \leq C \Delta t^{\gamma}
$$

for any fixed $\tau=n \Delta t \in[0, T]$, and $\Delta t$ sufficiently small.

Theorem 3.1 Under conditions (2.1)-(2.2), let $0<\theta<1,0<\Delta t<\min \left\{1, \frac{1}{4 \theta L_{\lambda}}, \frac{1}{\sqrt{K_{\lambda} \theta}}\right\}$, the continuous-time approximate solution $Y(t)$ defined by (3.4) will converge to the true solution of (2.5) in the mean square sense, i.e.,

$$
E \sup _{0 \leq t \leq T}|Y(t)-X(t)|^{2} \leq C_{5} \Delta t
$$

where $C_{5}$ is a positive constant independent of $\Delta t$.

Proof From (2.5) and (3.4), we have

$$
\begin{aligned}
Y(t) & -X(t) \\
= & \int_{0}^{t}(1-\theta)\left[f_{\lambda}\left(Z_{1}(s)\right)-f_{\lambda}\left(X\left(s^{-}\right)\right)\right]+\theta\left[f_{\lambda}\left(Z_{2}(s)\right)-f_{\lambda}\left(X\left(s^{-}\right)\right)\right] \mathrm{d} s \\
& \quad+\int_{0}^{t} g\left(Z_{2}(s)\right)-g\left(X\left(s^{-}\right)\right) \mathrm{d} W(s)+\int_{0}^{t} h\left(Z_{2}(s)\right)-g\left(X\left(s^{-}\right)\right) \mathrm{d} \tilde{N}(s) .
\end{aligned}
$$

For any $t_{1} \in[0, T]$, using the Cauchy-Schwarz inequality and the inequality $\mid \theta x+(1-$ $\theta)\left.y\right|^{2} \leq \theta|x|^{2}+(1-\theta)|y|^{2}$, we have

$$
\begin{aligned}
& E \sup _{0 \leq t \leq t_{1}}|Y(t)-X(t)|^{2} \\
& \quad \leq 3 E \sup _{0 \leq t \leq t_{1}} \mid \int_{0}^{t}(1-\theta)\left[f_{\lambda}\left(Z_{1}(s)\right)-f_{\lambda}\left(X\left(s^{-}\right)\right)\right]
\end{aligned}
$$




$$
\begin{aligned}
& +\left.\theta\left[f_{\lambda}\left(Z_{2}(s)\right)-f_{\lambda}\left(X\left(s^{-}\right)\right)\right] \mathrm{d} s\right|^{2} \\
& +3 E \sup _{0 \leq t \leq t_{1}}\left|\int_{0}^{t} g\left(Z_{2}(s)\right)-g\left(X\left(s^{-}\right)\right) \mathrm{d} W(s)\right|^{2} \\
& +3 E \sup _{0 \leq t \leq t_{1}}\left|\int_{0}^{t} h\left(Z_{2}(s)\right)-h\left(X\left(s^{-}\right)\right) \mathrm{d} \tilde{N}(s)\right|^{2} \\
& \leq 6 \sup _{0 \leq t \leq t_{1}} \int_{0}^{t} 1^{2} \mathrm{~d} s E \sup _{0 \leq t \leq t_{1}} \int_{0}^{t}\left|f_{\lambda}\left(Z_{1}(s)\right)-f_{\lambda}\left(X\left(s^{-}\right)\right)\right|^{2} \\
& +\left|f_{\lambda}\left(Z_{2}(s)\right)-f_{\lambda}\left(X\left(s^{-}\right)\right)\right|^{2} \mathrm{~d} s \\
& +3 E \sup _{0 \leq t \leq t_{1}}\left|\int_{0}^{t} g\left(Z_{2}(s)\right)-g\left(X\left(s^{-}\right)\right) \mathrm{d} W(s)\right|^{2} \\
& +3 E \sup _{0 \leq t \leq t_{1}}\left|\int_{0}^{t} h\left(Z_{2}(s)\right)-h\left(X\left(s^{-}\right)\right) \mathrm{d} \tilde{N}(s)\right|^{2} .
\end{aligned}
$$

Now using the Doob martingale inequality for the two martingale terms, we have

$$
\begin{aligned}
& E \sup _{0 \leq t_{\leq} t_{1}}|Y(t)-X(t)|^{2} \\
& \leq 6 t_{1} E \int_{0}^{t_{1}}\left|f_{\lambda}\left(Z_{1}(s)\right)-f_{\lambda}\left(X\left(s^{-}\right)\right)\right|^{2}+\left|f_{\lambda}\left(Z_{2}(s)\right)-f_{\lambda}\left(X\left(s^{-}\right)\right)\right|^{2} \mathrm{~d} s \\
& \quad+12 E\left|\int_{0}^{t_{1}} g\left(Z_{2}(s)\right)-g\left(X\left(s^{-}\right)\right) \mathrm{d} W(s)\right|^{2} \\
& \quad+12 E\left|\int_{0}^{t_{1}} h\left(Z_{2}(s)\right)-h\left(X\left(s^{-}\right)\right) \mathrm{d} \tilde{N}(s)\right|^{2} .
\end{aligned}
$$

Then Fubini's theorem and the martingale isometries give

$$
\begin{aligned}
E \sup _{0 \leq t \leq t_{1}}|Y(t)-X(t)|^{2} \\
\leq 6 T \int_{0}^{t_{1}} E\left|f_{\lambda}\left(Z_{1}(s)\right)-f_{\lambda}\left(X\left(s^{-}\right)\right)\right|^{2}+E\left|f_{\lambda}\left(Z_{2}(s)\right)-f_{\lambda}\left(X\left(s^{-}\right)\right)\right|^{2} \mathrm{~d} s \\
\quad+12 \int_{0}^{t_{1}} E\left|g\left(Z_{2}(s)\right)-g\left(X\left(s^{-}\right)\right)\right|^{2} \mathrm{~d} s \\
\quad+12 \lambda \int_{0}^{t_{1}} E\left|h\left(Z_{2}(s)\right)-h\left(X\left(s^{-}\right)\right)\right|^{2} \mathrm{~d} s
\end{aligned}
$$

Applying Lipschitz conditions (2.1) and (2.6), we get

$$
\begin{aligned}
E \sup _{0 \leq t \leq t_{1}}|Y(t)-X(t)|^{2} \\
\leq 6 T K_{\lambda} \int_{0}^{t_{1}} E\left|Z_{1}(s)-X\left(s^{-}\right)\right|^{2}+E\left|Z_{2}(s)-X\left(s^{-}\right)\right|^{2} \mathrm{~d} s \\
\quad+12 K \int_{0}^{t_{1}} E\left|Z_{2}(s)-X\left(s^{-}\right)\right|^{2} \mathrm{~d} s+12 \lambda K \int_{0}^{t_{1}} E\left|Z_{2}(s)-X\left(s^{-}\right)\right|^{2} \mathrm{~d} s
\end{aligned}
$$




$$
\begin{aligned}
= & 6 T K_{\lambda} \int_{0}^{t_{1}} E\left|Z_{1}(s)-X\left(s^{-}\right)\right|^{2} \mathrm{~d} s \\
& +6\left(T K_{\lambda}+2 K+2 \lambda K\right) \int_{0}^{t_{1}} E\left|Z_{2}(s)-X\left(s^{-}\right)\right|^{2} \mathrm{~d} s \\
\leq & 12 T K_{\lambda} \int_{0}^{t_{1}} E\left|Z_{1}(s)-Y\left(s^{-}\right)\right|^{2}+E\left|Y(s)-X\left(s^{-}\right)\right|^{2} \mathrm{~d} s \\
& +12\left(T K_{\lambda}+2 K+2 \lambda K\right) \int_{0}^{t_{1}} E\left|Z_{2}(s)-Y\left(s^{-}\right)\right|^{2}+E\left|Y(s)-X\left(s^{-}\right)\right|^{2} \mathrm{~d} s .
\end{aligned}
$$

Finally, applying Lemma 3.3, we have

$$
\begin{aligned}
& E \sup _{0 \leq t \leq t_{1}}|Y(t)-X(t)|^{2} \\
& \leq 12 T^{2} K_{\lambda} C_{3} \Delta t+12\left(T K_{\lambda}+2 K+2 \lambda K\right) T C_{4} \Delta t \\
&+12\left(T K_{\lambda}+T K_{\lambda}+2 K+2 \lambda K\right) \int_{0}^{t_{1}} E\left|Y(s)-X\left(s^{-}\right)\right|^{2} \mathrm{~d} s \\
& \leq 12 T^{2} K_{\lambda} C_{3} \Delta t+12\left(T K_{\lambda}+2 K+2 \lambda K\right) T C_{4} \Delta t \\
&+12\left(2 T K_{\lambda}+2 K+2 \lambda K\right) \int_{0}^{t_{1}} E \sup _{0 \leq r \leq s}\left|Y(r)-X\left(r^{-}\right)\right|^{2} \mathrm{~d} s .
\end{aligned}
$$

Using the Gronwall inequality (see [14]), we have

$$
E \sup _{0 \leq t \leq t_{1}}|Y(t)-X(t)|^{2} \leq C_{5} \Delta t
$$

Thus for any $t_{1} \in[0, T]$, we have

$$
E \sup _{0 \leq t \leq T}|Y(t)-X(t)|^{2} \leq C_{5} \Delta t
$$

\section{Mean-square stability}

In order to study the stability property of the $\operatorname{CSS} \theta$ method, we consider a linear test equation with scalar coefficients

$$
\mathrm{d} X(t)=a X\left(t^{-}\right) \mathrm{d} t+b X\left(t^{-}\right) \mathrm{d} W(t)+c X\left(t^{-}\right) \mathrm{d} N(t)
$$

where $a, b, c \in \mathbb{R}$. Hence, the mean-square stability of the zero solution to equation (4.1) was proved in [1], i.e.,

$$
\lim _{t \rightarrow \infty} E|X(t)|^{2}=0 \quad \Leftrightarrow \quad 2 a+b^{2}+\lambda c(c+2)<0 .
$$

Applying the CSS $\theta$ method (2.7)-(2.8) to equation (4.1), we have

$$
\begin{aligned}
& Y_{n}{ }^{*}=Y_{n}+\left[(1-\theta)(a+\lambda c) Y_{n}+\theta(a+\lambda c) Y_{n}{ }^{*}\right] h, \\
& Y_{n+1}=Y_{n}{ }^{*}+b Y_{n}{ }^{*} \Delta W_{n}+c Y_{n}{ }^{*} \Delta \tilde{N}_{n} .
\end{aligned}
$$


Definition 4.1 Under condition (4.2), a numerical method applied to equation (4.1) is said to be MS-stable if there exists $h_{0}(a, b, c, \lambda)>0$ such that the numerical solution sequence $Y_{n}$ produced by this numerical scheme satisfies

$$
\lim _{n \rightarrow \infty} E\left|Y_{n}\right|^{2}=0
$$

for all $h \in\left(0, h_{0}(a, b, c, \lambda)\right)$.

Theorem 4.1 Under condition (4.2), then for

$$
\Delta t \leq h_{0}(a, b, c, \lambda, \theta)=\frac{-B+\sqrt{B^{2}-4 A C}}{2 A},
$$

where

$$
\begin{aligned}
& A=(1-\theta)^{2}(a+\lambda c)^{2}\left(b^{2}+\lambda c^{2}\right), \\
& B=(1-2 \theta)(a+\lambda c)^{2}+2(1-\theta)(a+\lambda c)\left(b^{2}+\lambda c^{2}\right), \\
& C=2 a+b^{2}+\lambda c(c+2), \\
& \theta \in[0,1),
\end{aligned}
$$

the CSS $\theta$ method (2.7)-(2.8) applied to equation (4.1) is MS-stable.

Proof Assuming that $1-\theta(a+\lambda c) h \neq 0$, from (4.3) we have

$$
Y_{n}{ }^{*}=\frac{1+(1-\theta)(a+\lambda c) h}{1-\theta(a+\lambda c) h} Y_{n}
$$

Substituting this into (4.4) yields

$$
Y_{n+1}=\frac{1+(1-\theta)(a+\lambda c) h}{1-\theta(a+\lambda c) h}\left(1+b \Delta W_{n}+c \Delta \tilde{N}_{n}\right) Y_{n}
$$

Squaring both sides of (4.8), we can get

$$
\left|Y_{n+1}\right|^{2}=\left(\frac{1+(1-\theta)(a+\lambda c) h}{1-\theta(a+\lambda c) h}\right)^{2}\left(1+b \Delta W_{n}+c \Delta \tilde{N}_{n}\right)^{2}\left|Y_{n}\right|^{2}
$$

Noting that $E\left(\Delta W_{n}\right)=0, E\left[\left(\Delta W_{n}\right)^{2}\right]=h, E\left(\Delta \tilde{N}_{n}\right)=0, E\left[\left(\Delta \tilde{N}_{n}\right)^{2}\right]=\lambda h$, we have

$$
E\left|Y_{n+1}\right|^{2}=\left(\frac{1+(1-\theta)(a+\lambda c) h}{1-\theta(a+\lambda c) h}\right)^{2}\left(1+b^{2} h+\lambda c^{2} h\right) E\left|Y_{n}\right|^{2} .
$$

By the iteration of (4.10), we conclude that $\lim _{n \rightarrow \infty} E\left|Y_{n}\right|^{2}=0$ if

$$
\left(\frac{1+(1-\theta)(a+\lambda c) h}{1-\theta(a+\lambda c) h}\right)^{2}\left(1+b^{2} h+\lambda c^{2} h\right)<1,
$$


which is equivalent to

$$
(1+(1-\theta)(a+\lambda c) h)^{2}\left(1+b^{2} h+\lambda c^{2} h\right)<(1-\theta(a+\lambda c) h)^{2},
$$

i.e.,

$$
\begin{aligned}
& \left((1-\theta)^{2}(a+\lambda c)^{2}\left(b^{2}+\lambda c^{2}\right)\right) h^{2} \\
& \quad+\left[(1-2 \theta)(a+\lambda c)^{2}+2(1-\theta)(a+\lambda c)\left(b^{2}+\lambda c^{2}\right)\right] h \\
& \quad+2 a+b^{2}+\lambda c(c+2)<0 .
\end{aligned}
$$

Let

$$
\begin{aligned}
f(h)= & \left((1-\theta)^{2}(a+\lambda c)^{2}\left(b^{2}+\lambda c^{2}\right)\right) h^{2} \\
& +\left[(1-2 \theta)(a+\lambda c)^{2}+2(1-\theta)(a+\lambda c)\left(b^{2}+\lambda c^{2}\right)\right] h \\
& +2 a+b^{2}+\lambda c(c+2) .
\end{aligned}
$$

If $\theta=1$, (4.13) becomes

$$
-(a+\lambda c)^{2} h+2 a+b^{2}+\lambda c(c+2)<0 .
$$

By (4.2), we know that (4.15) holds for all $h>0$, i.e., the CSS $\theta$ method is MS-stable for all $h>0$. Note that if $\theta=1$, the $\operatorname{CSS} \theta$ method reduces to $\operatorname{CSSBE}$, and (4.15) coincides with Theorem 7 which was studied in [4].

If $\theta \in[0,1)$, let

$$
\begin{aligned}
& A=(1-\theta)^{2}(a+\lambda c)^{2}\left(b^{2}+\lambda c^{2}\right), \\
& B=(1-2 \theta)(a+\lambda c)^{2}+2(1-\theta)(a+\lambda c)\left(b^{2}+\lambda c^{2}\right), \\
& C=2 a+b^{2}+\lambda c(c+2) .
\end{aligned}
$$

In view of (4.2), we know that $a+\lambda c<0$, then $A \neq 0$ (if $A=0, b^{2}+\lambda c^{2}=0$, i.e., $b=0, c=0$, then equation (4.1) becomes nonsense), so we can get

$$
\begin{aligned}
A & >0, \\
B & =(1-2 \theta)(a+\lambda c)^{2}+2(1-\theta)(a+\lambda c)\left(b^{2}+\lambda c^{2}\right) \\
& <(1-2 \theta)(a+\lambda c)^{2}-2(1-\theta)(a+\lambda c)(2 a+2 \lambda c) \\
& =(-3+2 \theta)(a+\lambda c)^{2}<0, \\
C & <0, \\
\Delta & =B^{2}-4 A C>0 .
\end{aligned}
$$


So $f(h)=0$ has two real roots $h_{0}$ and $h_{1}$, with $h_{1}<0<h_{0}$, where

$$
\begin{aligned}
& h_{0}(a, b, c, \lambda, \theta)=\frac{-B+\sqrt{\Delta}}{2 A}>0, \\
& h_{1}(a, b, c, \lambda, \theta)=\frac{-B-\sqrt{\Delta}}{2 A}<0 .
\end{aligned}
$$

So we can easily obtain that $f(h)<0$ holds when

$$
h \in\left(0, h_{0}(a, b, c, \lambda, \theta)\right) .
$$

From (4.13), we know that the CSS $\theta$ method is MS-stable. This proves the theorem.

\section{Numerical experiments}

We consider the following equation:

$$
\left\{\begin{array}{l}
\mathrm{d} X(t)=a X\left(t^{-}\right) \mathrm{d} t+b X\left(t^{-}\right) \mathrm{d} W(t)+c X\left(t^{-}\right) \mathrm{d} N(t) \\
X(0)=1
\end{array}\right.
$$

Equation (5.1) has the exact solution

$$
X(t)=X(0) \exp \left(\left(a-\frac{1}{2} b^{2}\right) t+b W(t)\right)(1+c)^{N(t)}
$$

see, for example, [15].

To illustrate the convergence order and the linear mean-square stability of the CSS $\theta$ method, we choose the following examples from the reference [7].

Example $5.1 a=-7, b=1, c=1, \lambda=4$.

Example 5.2 $a=2, b=2, c=-0.9, \lambda=9$.

In this section, the data used in all figures are obtained by the mean square of data by 1,000 trajectories, that is, $\omega_{i}: 1 \leq i \leq 1,000, Y_{n}=1 / 1,000 \sum_{i=1}^{1,000}\left|Y_{n}\left(\omega_{i}\right)\right|^{2}$; in all figures $t_{n}$ denotes the mesh-point.

To show the strong convergence order of the $\operatorname{CSS} \theta$ method, we apply the $\operatorname{CSS} \theta$ method to Example 5.1. First, we plot the exact solution of Example 5.1 for one sample path and the $\operatorname{CSS} \theta$ approximations in Figure 1. Then we simulate the numerical solutions with five different step sizes $h=2^{p-1} \Delta t$ for $1 \leq p \leq 5, \Delta t=2^{-14}$. The mean-square errors $\varepsilon=1 / 1,000 \sum_{i=1}^{1,000}\left|Y_{n}\left(\omega_{i}\right)-X(T)\right|^{2}$ all measured at time $T=1$ are estimated by trajectory averaging. We plot our approximation to $\sqrt{\epsilon}$ against $\Delta t$ on a log-log scale. For reference a dashed line of slope one-half is added. We see that the slopes of the two curves appear to match well in Figure 2. Hence, our results are consistent with a strong order of convergence equal to $1 / 2$.

To illustrate the step size $h$ on the mean-square stability of the CSS $\theta$ method, we applied the $\operatorname{CSS} \theta$ method to Examples 5.1 and 5.2. 

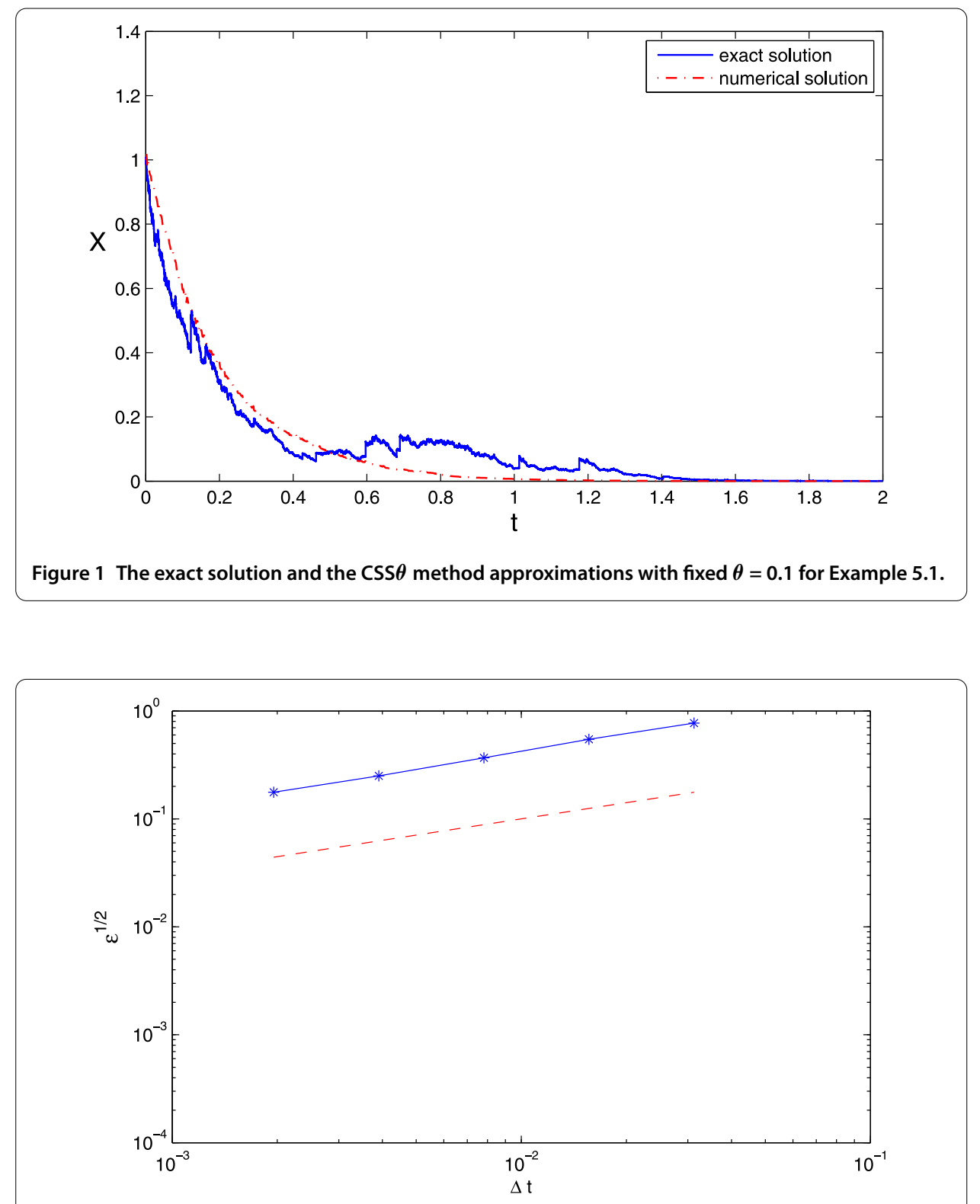

Figure 2 The convergence rate of the $\operatorname{css} \theta$ method for Example 5.1 with fixed $\theta=0.2$.

For Example 5.1, we first choose $\theta=0.1$, then by Theorem 4.1 we know that the CSS $\theta$ method is MS-stable when $h_{0}(a, b, c, \lambda, \theta)=0.5897$. Figure 3 illustrates the numerical solution produced by the CSS $\theta$ method is MS-stable when $h=1 / 2$. However, applied to the same test equation, and also choose $\theta=0.1$, then by Theorem 3.1 in [7] the CSTM is MSstable when the step size $h \in(0,0.138)$.

When we choose $\theta=0.4$, by Theorem 4.1 we know that the CSS $\theta$ method is MS-stable when $h_{0}(a, b, c, \lambda, \theta)=1.0583$, while the CST method in [7] is MS-stable when the step size $h \in(0,0.556)$. Figure 4 illustrates the numerical solution produced by the CSS $\theta$ method is MS-stable when $h=1$. At the same times we know that the Euler-Maruyama (EM) method in [1] is MS-stable for Example 5.1 when the step size $h \in(0,0.111)$. 


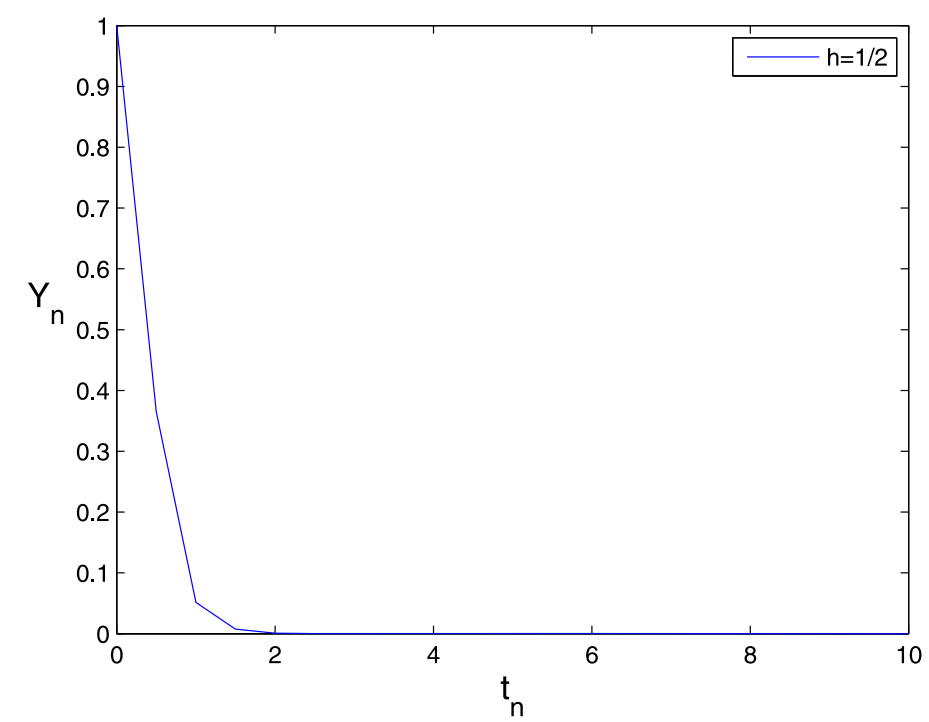

Figure 3 The $\operatorname{CSS} \theta$ method approximations for Example 5.1 with $\theta=0.1, h=1 / 2$.

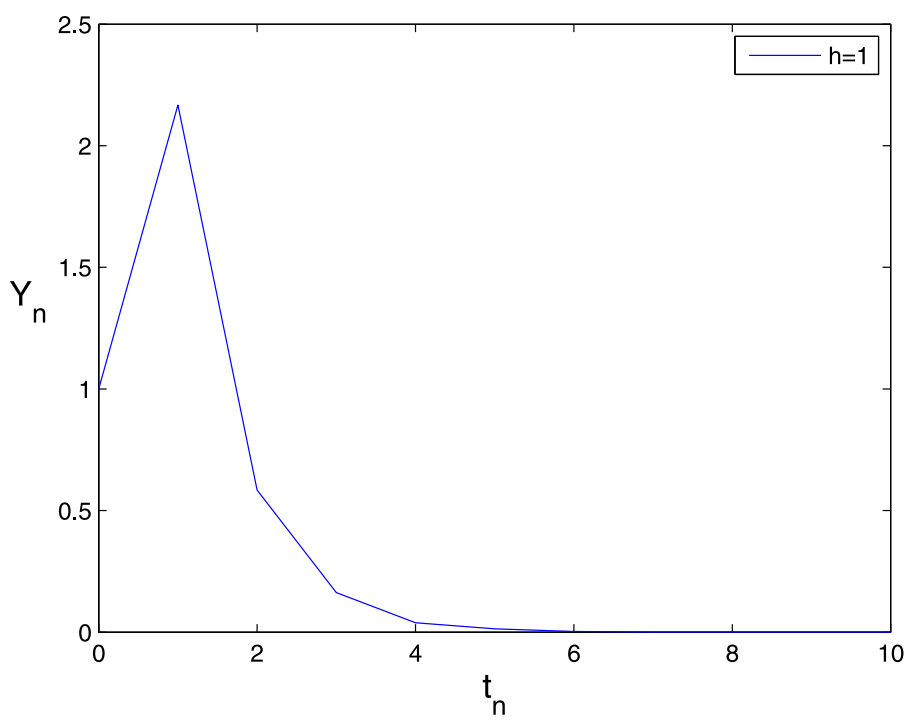

Figure 4 The $\operatorname{CSS} \theta$ method approximations for Example 5.1 with $\theta=0.4, h=1$.

Remark 1 Figures 3 and 4 indicate that the restriction on the step size $h$ of the CSS $\theta$ method for the MS-stability is less than that of both the CST method and the EM method.

For Example 5.2, we note that $c=-0.9<0$, then the theta method in [1] is not guaranteed to preserve stability for all $\Delta t \geq 0$. However, if we choose $\theta=0.1$, then by Theorem 4.1 we know that the CSS $\theta$ method is MS-stable when $h_{0}(a, b, c, \lambda, \theta)=0.2862$, and when $\theta=0.4, h_{0}(a, b, c, \lambda, \theta)=0.5091$. Figure 5 and Figure 6 (upper) illustrate the numerical solution produced by the CSS $\theta$ method is MS-stable for Example 5.2 when the step size $h \in\left(0, h_{0}(a, b, c, \lambda, \theta)\right)=(0,0.5091)$. 


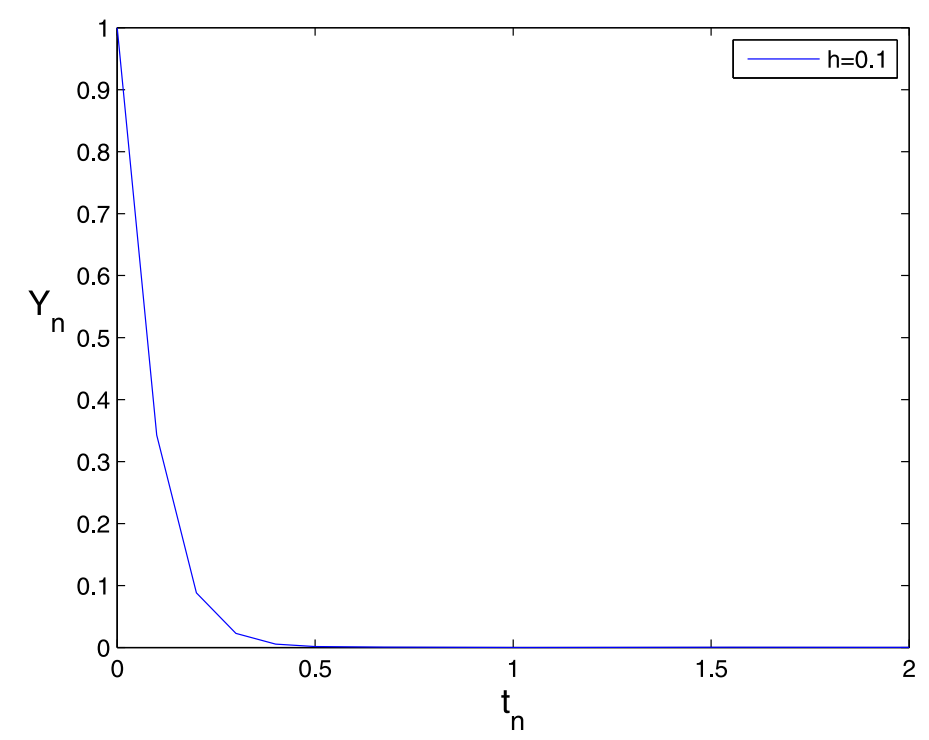

Figure 5 The $\operatorname{CSS} \theta$ method approximations for Example 5.2 with $\theta=0.1, h=0.1$.
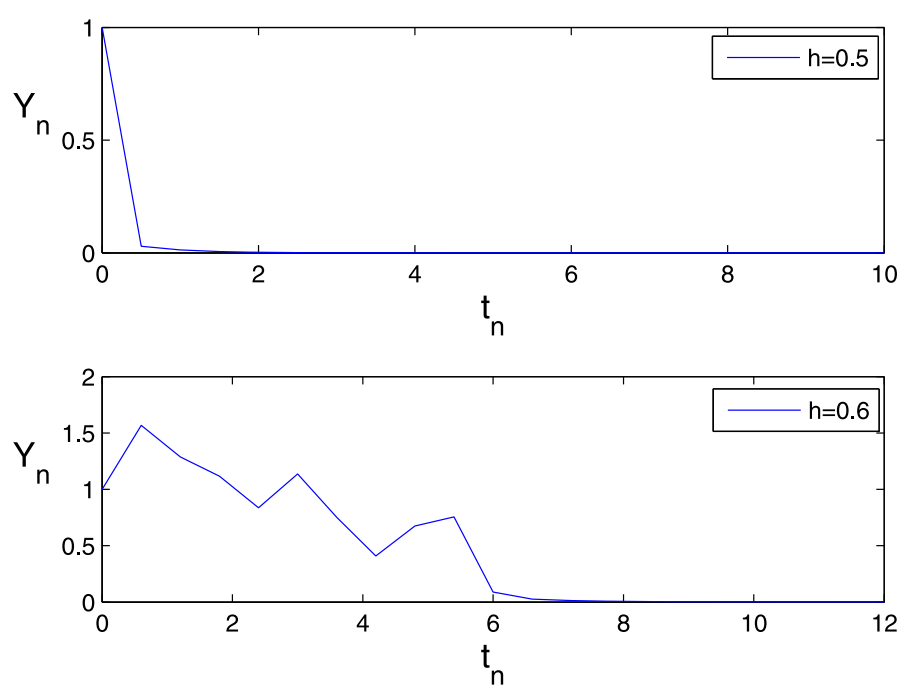

Figure 6 The $\operatorname{CSS} \theta$ method approximations for Example 5.2 with $\theta=0.4, h=0.5$ (upper), $h=0.6$ (lower).

At last, Figure 6 (lower) shows that the numerical solution of the $\operatorname{CSS} \theta$ method is still stable when $h=0.6>h_{0}(a, b, c, \lambda, \theta)=0.5091$. This implies that maybe the mean-square stability bound we obtained by Theorem 4.1 is not optimal.

Competing interests

The authors declare that they have no competing interests.

Authors' contributions

All the authors contributed equally to this work. They all read and approved the final version of the manuscript.

\section{Author details}

${ }^{1}$ Department of Mathematics, Tianjin Polytechnic University, Tianjin, 300387, P.R. China. ${ }^{2}$ College of Basic Science, Tianjin Agricultural University, Tianjin, 300384, P.R. China. 


\section{Acknowledgements}

This research was supported with funds provided by the National Natural Science Foundation of China (Nos. 11226321, 11272229 and 11102132). We thank two anonymous reviewers for their very valuable comments and helpful suggestions which improved this paper significantly.

Received: 27 December 2013 Accepted: 8 July 2014 Published: 04 Aug 2014

\section{References}

1. Higham, DJ, Kloeden, PE: Convergence and stability of implicit methods for jump-diffusion. Int. J. Numer. Anal. Model. 3, 125-140 (2006)

2. Higham, DJ, Kloeden, PE: Strong convergence rates for backward Euler on a class of nonlinear jump-diffusion problems. J. Comput. Appl. Math. 205, 949-956 (2007)

3. Chalmers, GD, Higham, DJ: Convergence and stability analysis for implicit simulations of stochastic differential equations with random jump magnitudes. Discrete Contin. Dyn. Syst., Ser. B 9, 47-64 (2008)

4. Higham, DJ, Kloeden, PE: Numerical methods for nonlinear stochastic differential equations with jumps. Numer. Math. 101, 101-119 (2005)

5. Bruti-Liberati, N, Platen, E: On the weak approximation of jump-diffusion processes. Technical report, University of Technology Sydney, Sydney (2006)

6. Bruti-Liberati, N, Platen, E: Strong approximations of stochastic differential equations with jumps. J. Comput. Appl. Math. 205, 982-1001 (2007)

7. Wang, XJ, Gan, SQ: Compensated stochastic theta methods for stochastic differential equations with jumps. Appl. Numer. Math. 60, 877-887 (2010)

8. Hu, L, Gan, SQ: Convergence and stability of the balanced methods for stochastic differential equations with jumps. Int. J. Comput. Math. 88, 2089-2108 (2011)

9. Ding, XH, Ma, Q, Zhang, L: Convergence and stability of the split-step $\theta$-method for stochastic differential equations. Comput. Math. Appl. 60, 1310-1321 (2010)

10. Gikhman, II, Skorokhod, AV: Stochastic Differential Equations. Springer, Berlin (1972)

11. Sobczyk, K: Stochastic Differential Equations with Applications to Physics and Engineering. Kluwer Academic, Dordrecht (1991)

12. Smart, DR: Fixed Point Theorems. Cambridge University Press, Cambridge (1974)

13. Gardon, A: The order of approximation for solutions of Itô-type stochastic differential equations with jumps. Stoch. Anal. Appl. 22, 679-699 (2004)

14. Mao, XR: Stochastic Differential Equations and Applications. Ellis Horwood, Chichester (1997)

15. Glasserman, P: Monte Carlo Methods in Financial Engineering. Springer, Berlin (2003)

10.1186/1687-1847-2014-209

Cite this article as: Tan et al.: Convergence and stability of the compensated split-step $\theta$-method for stochastic differential equations with jumps. Advances in Difference Equations 2014, 2014:209

\section{Submit your manuscript to a SpringerOpen ${ }^{\circ}$ journal and benefit from:}

- Convenient online submission

- Rigorous peer review

- Immediate publication on acceptance

- Open access: articles freely available online

- High visibility within the field

- Retaining the copyright to your article 